Zsuzsanna Buzás ${ }^{{ }^{*}}$ Huan-Tsung Chang ${ }^{1 * \star}$

Nancy E. Vieira ${ }^{2}$

Alfred L. Yergey ${ }^{2}$

Miroslava Stastna $^{1 \star \star \star}$

Andreas Chrambach ${ }^{1}$

${ }^{1}$ Section on Macromolecular

Analysis, and

2Section on Mass Spectrometry and Metabolism, Laboratory of Cellular and Molecular Biophysics, NICHD, NIH, Bethesda, MD, USA

\section{Direct vertical electroelution of protein from a PhastSystem band for mass spectrometric identification at the level of a few picomoles}

\begin{abstract}
An electroelution apparatus prototype of a new design was constructed. In that design, the electric field passes vertically through the protein band located on a horizontal (PhastSystem) minigel polymerized on a net of Gel-Fix (Serva). A simple, home-made apparatus allows for electroelution of protein bands at the level of a few picomoles and their identification, after concentration, by matrix-assisted laser desorption/ionizationtime of flight mass spectrometry. The technique is applicable to one-dimensional (1-D) or two-dimensional (2-D) gels of any size, but has been exemplified only by application to 1-D minigels to demonstrate the lower limits of protein load of the method. When in the course of further development of the prototype it will be combined with a modification to two dimensions of the electroelution mechanism under computer control of the high-performance gel electrophoresis apparatus ${ }^{\star \star \star \star}$ (formerly of Lablntelligence), the new design appears uniquely qualified for an automated spot elution from 2-D gels under avoidance of gel sectioning.
\end{abstract}

Keywords: Vertical electroelution / Picomolar protein identification / Mass spectrometry

PRO 0051

\section{Introduction}

This paper deals with the transfer of protein under a gel electrophoretic band (spot) to mass spectrometry with the purpose of identifying the protein. The prevalent approach to such mass spectrometric identification of protein spots on gel electropherograms employs excision of the gel band followed by in situ proteolysis of the spot and extraction of the peptides by either diffusion or electrophoresis [1-3]. The present approach differs from the conventional one in two major ways: (i) The intact protein is extracted by electroelution, tested for its homogeneity and identified by its intact mass, independently of whether such identification is followed later by proteolysis and identification of the protein on the basis of its peptide map; (ii) The electroelution is performed on the electropherogram directly, i.e. without slicing of the gel. The advantages of these two methodological differences over the conventional technique of protein transfer from gel electropherograms to MS will be addressed in Section 4.

The direct electroelution of intact proteins and their transfer into mass spectrometry (MS) are not original. Direct electroelution, without gel sectioning, has been achieved

Correspondence: Dr. A. Chrambach, Bldg. 10, Rm. 9D50, NIH, Bethesda, MD 20892-1580, USA

E-mail: acc@cu.nih.gov

Fax: +1-301-402-0263

Abbreviation: HPGE, high-performance gel electrophoresis under computer control by use of the automated 1-D electrophoresis high-performance gel electrophoresis (HPGE) apparatus [4]. Thereby isolated intact single proteins have been transferred to and identified by MS [5-8]. Direct electroelution and MS identification of peptides has also been reported [9]. Sequential transfer to MS of electroeluates of more than a single protein covalently prelabeled with a fluorophore has been possible [8] albeit with lowered sensitivity of identification by peptide map. Simultaneous electroelution and transfer to MS of multiple species of intact proteins constituting a gel electrophoretic protein pattern has also been reported [e.g. $10,11]$. A procedure for the testing of protein homogeneity prior to MS is original to the present work. Its second original feature, relevant to its potential for automation, lies in the orientation of the electric field, vertically passing the peak distribution.

\section{Materials and methods}

\subsection{Protein}

The SDS protein sample was the low molecular weight calibration kit (Pharmacia, Uppsala, Sweden Cat. No.

* Agricultural Biotechnology Center, Gödöllö, Hungary

** Department of Chemistry, National Taiwan University, Taipei, Taiwan

*** On leave from the Institute of Analytical Chemistry, Czech Academy of Sciences, Brno, Czech Republic

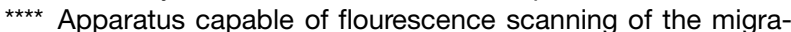
tion path and computer directed electroelution of bands. 
Table 1. Discontinuous buffer system for SDS-PAGE, $25^{\circ} \mathrm{C}$

\begin{tabular}{llllll}
\hline & \multicolumn{2}{l}{ Stacking gel buffer } & \multicolumn{2}{l}{ Resolving gel buffer } & \multicolumn{2}{l}{$\begin{array}{l}\text { Catholyte } \\
\text { and anolyte } \\
\end{array}$} & $\begin{array}{lllll}\text { As set } \\
(\mathrm{M})\end{array}$ & $\begin{array}{l}\text { Operative } \\
(\mathrm{M})\end{array}$ & $\begin{array}{l}\text { As set } \\
(\mathrm{M})\end{array}$ & $\begin{array}{l}\text { Operative } \\
(\mathrm{M})\end{array}$ & $\begin{array}{l}\text { (M) } \\
\text { Acetic acid }\end{array}$ & 0.1120 & & 0.1120 & & \\
$\begin{array}{l}\text { Tricine } \\
\text { Tris }\end{array}$ & 0.1120 & 0.0831 & & 0.0831 & 0.2000 \\
$\mathrm{pH}$ & 6.39 & 8.06 & 6.39 & 8.06 & \\
lonic strength & 0.1097 & 0.0420 & 0.1097 & 0.0420 & \\
$\mathrm{RM}^{\mathrm{a})}$ (min) & & 0.212 & & & \\
$\mathrm{RM}$ (max) & 0.783 & & & & \\
\hline
\end{tabular}

a) $\mathrm{RM}=$ net mobility of an analyte (e.g. a protein), expressed relative to the mobility of $\mathrm{Na}^{+}$

$\mathrm{RM}(\min )=$ minimal mobility of an analyte to migrate within the stack

$\mathrm{RM}(\max )=$ maximal mobility of an analyte to migrate within the stack

stack = designation of a system of moving boundaries migrating at the same velocity (isotachophoretically) dictated by a regulation function

17-0446-01). Proteins ranging in molecular mass from 14.4 to $97.0 \mathrm{kDa}$ were dissolved in $0.2 \mathrm{~mL}$ (for obtaining a Coomassie Blue stained electropherogram of $1 \mu \mathrm{L}$ of the mixture) or $33.2 \mu \mathrm{L}$ (for electroelution of the BSA band from the electropherogram of $1 \mu \mathrm{L}$ of the mixture) of $10 \mathrm{~mm}$ Tris- $\mathrm{HCl}, 2 \% \mathrm{SDS}, 5 \% \quad \beta$-mercaptoethanol, $0.01 \%$ bromophenol blue, 1 mm EDTA, pH 8.0.

\subsection{Polyacrylamide gel electrophoresis}

PAGE was carried out in the PhastSystem (AmershamPharmacia, Piscataway NJ, USA Cat.No. 18-1018-23) with gels of $12 \% \mathrm{~T}, 2 \% \mathrm{C}$ (Bis), polymerized on Gel-Fix (Serva, Heidelberg, Germany, Cat. No. 42858) using the procedure described previously (Section 2.5 of [12]); the mechanical stability of the Gel-Fix was strengthened by bonding of GelBond-PAG (BMA, Rockland, ME, USA, Cat. No. 54727) to it, with its hydrophobic side resting on the Gel-Fix. The discontinuous buffer system used (Table 1) was that of the PhastSystem SDS-PAGE evaluated by the program of Jovin (Appendix 3 of [13]). The stacking gel was $6 \% \mathrm{~T}, 3 \% \mathrm{C}$ (Bis). The lengths of resolving gel, stacking gel and catholyte strip were those of the commercial PhastSystem gels. Electrophoresis was carried out at $15^{\circ} \mathrm{C}$ with current regulation at $10 \mathrm{~mA}$ as specified in PhastSystem File No. 111, until the tracking dye, bromophenol blue, approached the anodic terminus of the gel (in about 25 min). Protein bands were stained with the GelCode E-Zinc Reversible Stain Kit (Pierce, Rockford IL, USA, Cat. No. 24582). The gel on its GelFix and GelBond support was immersed in $5 \mathrm{~mL}$ E-Zinc stain solution for $10 \mathrm{~min}$. After removal of the GelBond from the Gel-Fix supported gel, it was placed onto the black hard-rubber sheet cover (lined with Parafilm) of the apparatus (Fig. 1). The gel was submerged under E-Zinc Developer for $0.5 \mathrm{~min}$. The Zn-fixed transparent protein bands, visualized against an opaque background, were marked by black marking pen (Pilot ultra fine point, permanent). The gel was washed with water and subsequently treated for $2 \mathrm{~min}$ with an EDTA solution (E-Zinc Eraser) (which solubilizes the protein $\mathrm{Zn}$-precipitates), using $5 \mathrm{~mL}$ to cover the entire gel surface.

\subsection{Electroelution}

The gel on its Gel-Fix support was covered by and equilibrated against $25 \mathrm{~mm}$ Tris, $192 \mathrm{~mm}$ glycine, $50 \mathrm{~mm}$ EDTA, $\mathrm{pH} 8.3$ for 2 min and transferred onto the black rubber top of the catholyte reservoir of the electroelution apparatus, a plastic pipet tip box $(12.4 \times 7.8 \times 5.0 \mathrm{~cm})($ Fig. 1$)$. The rubber sheet is slit at its center for insertion of a wick, and contains laterally a hole for insertion of the cathode. The wick is a strip of $0.4 \times 5 \mathrm{~cm}$ of blotting paper (Blot absorbent filter paper, extra thick, Bio-Rad, Hercules CA, USA Cat. No. 170-3965) saturated with catholyte. The gel resting on the sheet remains covered with catholyte at all times. The anolyte tube (Beckman Centrifuge tube, ultra-clear, $0.5 \times 1$ inch, Cat. No. 344057) was half-filled with $1 \%$ agarose (IsoGel, BMA, Cat. No. 50202) gelled in anolyte at the side opposite from the elution tube. The other half of the anolyte tube was filled with anolyte. The elution tube forming the electroeluate chamber (a glass tube of $5 \mathrm{~cm}$ length and rectangular cross-section, 


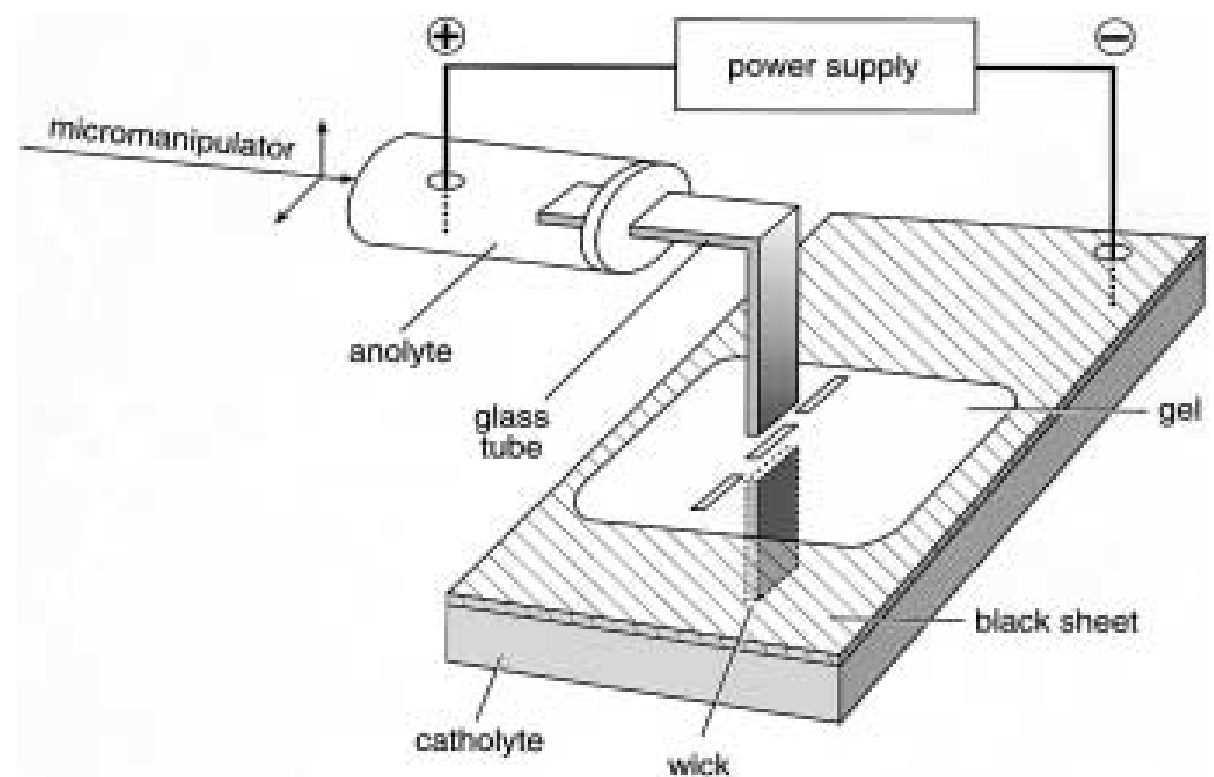

Figure 1. Apparatus for direct electroelution with a vertical field orientation of proteins from gel electrophoretic bands: (i) Catholyte reservoir, a plastic pipet tip box (approx $13 \times 8 \mathrm{~cm}$ in surface area) with a (ii) black hard rubber cover with a slit $(4 \times 1 \mathrm{~mm})$ and an entrance hole for the cathode; (iii) filter paper wick of $5 \mathrm{~cm}$ length, $4 \times 1 \mathrm{~mm}$ cross-sectional area; (iv) glass tube of $5 \mathrm{~cm}$ length, $0.4 \times 4 \mathrm{~cm}$ cross-sectional area, bent at a right angle at $3 \mathrm{~cm}$ length; (v) anolyte reservoir, a plastic centrifuge tube with a hole for entrance of the anode; one half of the tube, at the side opposite to the glass tube, is filled with agarose gel in anolyte; (vi) the anolyte reservoir is clamped to a micromanipulator; the glass tube, inserted through the cap of the anolyte reservoir, is positioned over the band by the micromanipulator.

$0.4 \mathrm{~mm} \times 4.0 \mathrm{~mm}$ I.D. (VitroCom Inc., Rockaway NJ, USA Cat. No. 2540), bent at a right angle at $3 \mathrm{~cm}$, is pushed into and held by a slitted rubber cap fitting the anolyte tube (Vial Cap, Beckman, Cat. No. 359079). Anolyte is allowed to pass through the elution tube until flow ceases. The anode is inserted into the agarose-anolyte through a hole (4 $\mathrm{mm}$ diameter) in the anolyte reservoir tube (Fig. 1). The elution tube (resting with its shorter leg in the anolyte reservoir) is then positioned over the band, using the micromanipulator (Narishige, Japan, Type GJ). Starting immediately after lowering the elution tube onto the band, bands were electroeluted into it for a duration of $3 \mathrm{~min}$ at $210 \mathrm{~V}(4.5 \mathrm{~mA})$ at room temperature. The elution tube was gently extruded from the anolyte tube and its cap, and a $1 \mathrm{~mL}$ syringe filled with anolyte was slowly discharged into the short leg of the elution tube to exert enough pressure for overcoming the capillary adhesion and start the flow of the electroeluate into a polypropylene microtube (Sarstedt, Newton, NC, USA, Cat. No. 72 699).

\subsection{Concentration and desalting of the electroeluate}

The electroeluate of approximately $100 \mu \mathrm{L}$ was concentrated to a final volume of $5 \mu \mathrm{L}$ by ultrafiltration (Microcon YM-10, Millipore, Bedford, MA, USA, Cat. No. 42406) and centrifugation at $10000 \mathrm{~g}$. For the purpose of desalting, $100 \mu \mathrm{L}$ of water were added to the ultrafiltrate and sedimentation was repeated. This was done twice.

\subsection{Re-electrophoresis of the electroeluate by PhastSystem SDS-PAGE and CZE}

A $1 \mu \mathrm{L}$ aliquot of the ultrafiltrate was analyzed by SDSPAGE, using an 8-25\%T PhastSystem pore gradient gel and automated Coomassie Blue staining. Another aliquot was used for CZE analysis. CZE was carried out in the P/ACE-2200 apparatus (Beckman, Fullerton, CA, USA), equipped with UV detector. A fused silica capillary of $100 \mu \mathrm{m}$ ID and $375 \mu \mathrm{m}$ OD with length of $27 \mathrm{~cm}$ externally coated with polyimide (Polymicro Technologies, Phoenix AZ, USA), was used. The detection window was created at $20 \mathrm{~cm}$ measured from the injection (cathodic) end, by applying a glowing wire to remove the external coating. Thereafter, the capillary was internally coated by linear polyacrylamide as described by Hjerten [14] to suppress the electroosmotic flow. Electroosmotic flow was measured prior to use of each capillary by determining the time of displacement of buffer $(0.05 \mathrm{M}$ Tris, $0.025 \mathrm{M}$ CHES, pH 9.0) containing $0.05 \%$ mesityl oxide (Aldrich, Milwaukee, WI, USA, Cat. No. 141-79-7) from the anodic reservoir to the detector. Capillaries exhibiting electroosmosis in excess of $1 \times 10^{-5} \mathrm{~cm}^{2} / \mathrm{sV}$ were discarded. The sample was pressure injected for $5 \mathrm{~s}$, a BSA standard $(2 \mathrm{mg} / \mathrm{mL})$ for $1 \mathrm{~s}$. CZE was conducted as described [15] at a field strength of $370 \mathrm{~V} / \mathrm{cm}$. The electric current did not exceed $12 \mu \mathrm{A}$. The UV detection at $214 \mathrm{~nm}$ was used.

\subsection{MALDI-TOF-MS of the electroeluate}

MALDI-TOF-MS analysis was performed using a PerSeptive BioSystems Voyager Elite DE-STR (PE-Bio- 
Electroelution

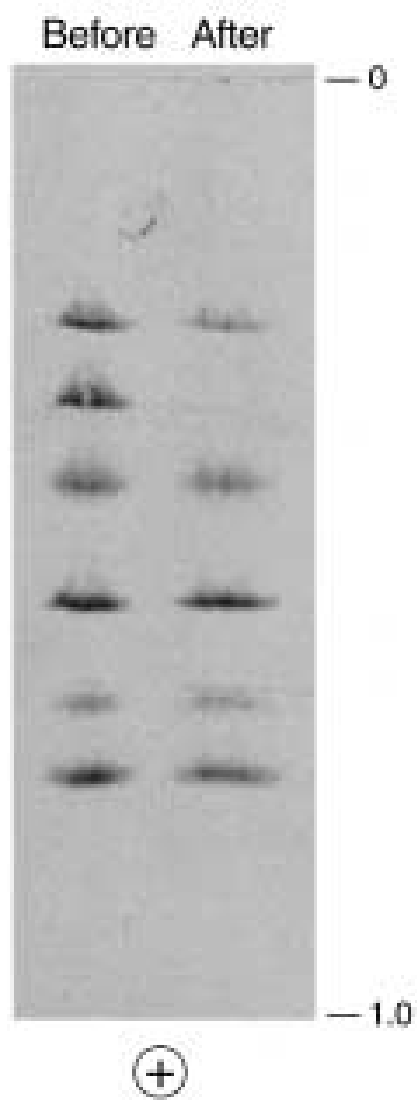

Figure 2. PhastSystem SDS-PAGE electropherogram of the mixture of six standard proteins before and after electroelution of the BSA band. PhastSystem 8-25\%T pore gradient gel, operative $\mathrm{pH} 8.06$, ionic strength $0.042,15^{\circ} \mathrm{C}$.

systems, Framingham, MA, USA) MALDI-TOF mass spectrometer. The instrument was operated in linear mode at $25 \mathrm{kV}$ accelerating voltage and $300 \mathrm{nsec}$ ion extraction delay. Samples were applied to the plate as follows. First, a $0.5 \mu \mathrm{L}$ aliquot of matrix, a saturated solution of sinnapinic acid in acetone, was applied to the plate and allowed to dry. Then an equal volume of the protein sample provided the second layer and was also allowed to dry in air. Finally, an additional $0.5 \mu \mathrm{L}$ aliquot of matrix, sinnapinic acid in $1: 1 \mathrm{CH}_{3} \mathrm{CN}: 0.1 \%$ TFA, was applied over each sample.

\section{Results}

\subsection{Apparatus design for the direct protein electroelution from gel bands}

The design presented in this paper (Fig. 1) features four advances: (i) The orientation of the electric field is entirely vertical to the direction of migration (Fig. 5B of [16]) rather than one which bends at a right angle in its passage through the peak [Fig. 5A of [16]); thus, the entire band area, inclusive of ascending and descending limbs, is electroeluted, rather than only the trailing half of the peak; (ii) The vertical orientation of the electric field has been achieved through use of a gel-adhesive network, Gel-Fix (Serva), in lieu of the previously used current-impermeable gel supports; it was shown experimentally that Gel-Fix did not resist the current flow. On gel electrophoresis through two identical cylindrical gels, one sealed with Gel-Fix at the anodic end, the other unsealed, the voltage at the same regulated current was indistinguishable (Li, Y.-M., personal communication); (iii) The protein load is downscaled to the level of a few picomoles through application of the electroelution to the slide-sized, $0.45 \mathrm{~mm}$ thick PhastSystem minigels; (iv) The design, unlike the previous ones reported from this laboratory (see Section 1) lends itself to the sequential electroelution of multiple bands.

\subsection{Electroelution of a protein band derived from a load of $2.5 \mu \mathrm{g}$ of BSA on a PhastSystem SDS-PAGE gel}

Six proteins of a mixture of size standards ranging in molecular mass from 14 to $97 \mathrm{kDa}$ were separated by SDS-PAGE on the PhastSystem (Fig. 2, left). The $67 \mathrm{kDa}$ protein band of BSA was electroeluted in presence of the five bands of the other proteins after detection of the gel pattern by $\mathrm{Zn}$-staining. A parallel lane on the identical gel used for the electroelution of the BSA band was subsequently stained with Coomassie Blue. It shows the disappearance of the $67 \mathrm{kDa}$ band due to successful electroelution (Fig. 2). The Coomassie Blue stained gel pattern of the concentrated electroeluate of the BSA band and its CZE pattern, respectively, are shown in Fig. 3.

\subsection{MALDI-TOF analysis of the electroeluate}

An aliquot of $0.5 \mu \mathrm{L}$ of the concentrated electroeluate containing less than 3.75 picomoles (in view of less than $100 \%$ recovery of the protein in the electroeluate) of the $67 \mathrm{kDa}$ protein was identified as BSA by MALDI-TOF-MS by the agreement of its $\mathrm{m} / \mathrm{z}$ ratio of 66,952 for the $\mathrm{MH}^{+}$peak and of 33,698 for the $\mathrm{MH}^{2+}$ peak with a BSA standard (Fig. 4).

\section{Discussion and conclusion}

\subsection{Direct electroelution vs protein recovery requiring gel slicing}

Electroelution of proteins from bands (spots) on the intact gel electropherogram has been applied to 1-D gels for a number of years prior to this study [4, 17-19]. Linked to fluorescent prelabeling of the protein (ibid)., it has the 


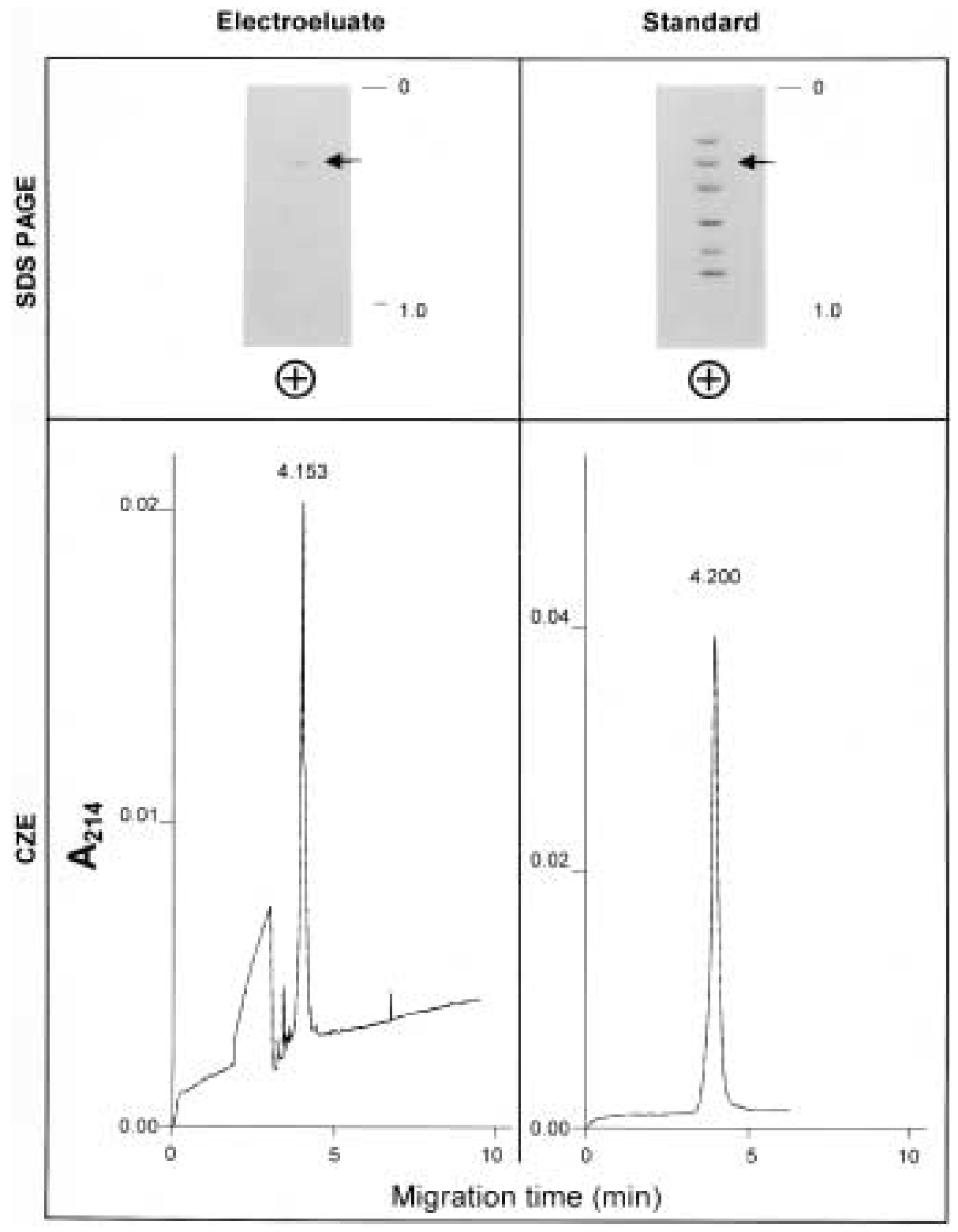

Figure 3. Electropherograms of the electroeluate of the BSA band (obtained as shown in Fig. 2) after concentration by ultrafiltration and of a BSA standard, using a 8-25\%T pore gradient SDS-PAGE gel (PhastSystem) (top panels) and capillary zone electrophoresis (CZE) (bottom panels). advantage of avoiding fixation of the protein; thereby solubility is maintained and recovery improved. The procedure of recovery is less labor intensive and faster than that based on band excision followed by elution of the protein or its proteolytic products. Potentially, it is more compatible with automation of the recovery.

\subsection{Recovery of intact protein vs that of its proteolytic products}

Direct electroelution has been applied to the transfer of intact protein from gel electropherograms to MS [5 to 8, and the present study]. This has the advantage that the homogeneity of the protein can be tested prior to MS of the intact protein for identification by its mass or of its proteolytic products for identification on the basis of its peptide map. It has the disadvantages of requiring a higher gel electrophoretic load than the transfer to MS of the products of in situ proteolysis. Also, the solubility of the intact SDS protein is likely to be less than that of its proteolytically formed peptides.

\subsection{Vertical vs orthogonal electroelution}

Previous electroelution of proteins by use of the (commercially no more available) HPGE apparatus [4] employed an electric field passing orthogonally through the band distribution (Fig. 5A of [16]) while the present design of electro- 


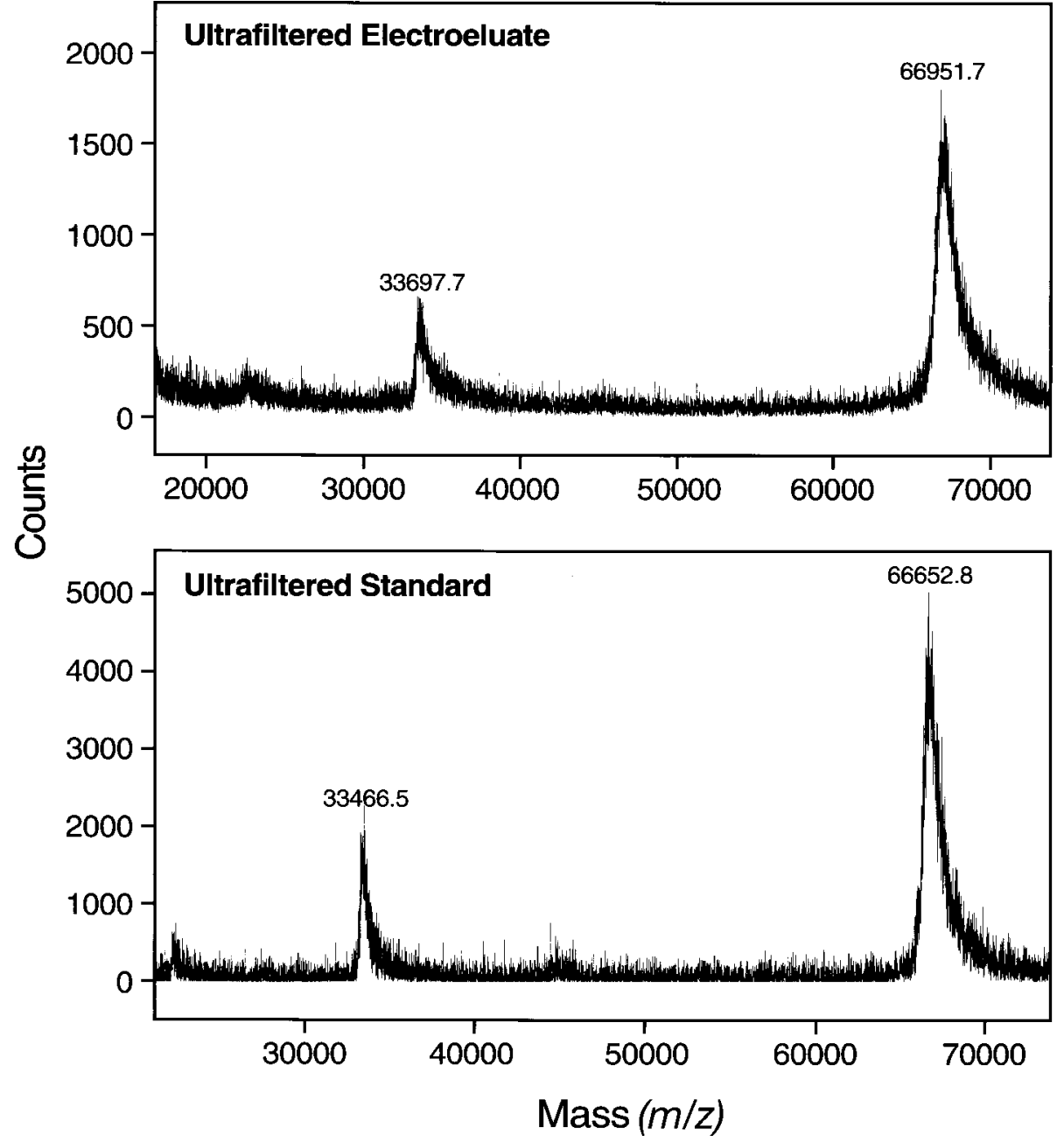

Figure 4. MALDI-TOF mass spectra of $10 \%$ (3.7 picomoles) of the electroeluate derived from a gel electrophoresis load of $2.5 \mu \mathrm{g}$ of the band of BSA and of a 1 picomole sample of BSA, both subjected to concentration by ultrafiltration under identical conditions. elution employs an electric field passing vertically through the band distribution (Fig. 5B of [16]). Vertical electroelution, i.e. that through the mode of the peak distribution, rests on the finding that a network bonded to polyacrylamide gel (GelFix, Serva) is freely permeable to the electric current. The advantages of the vertical orientation of electroelution are twofold: (i) The electroelution proceeds symmetrically across the entire band distribution and comprises the mode of that distribution, by contrast with the previous orthogonal field orientation which led to a loss of the protein along the ascending limb of the distribution and restricted the order of sequential band elution to that proceeding from the slowest to the fastest migrating proteins [20]. (ii) Electroelution of one spot becomes independent of the electroelution of any other spot. That fact allows for sequential electroelution of bands on a single gel electropherogram which the previously used orthogonal field orientation interfered with. The sequential electroelution is important since it is a necessary condition for a later application of the procedure to 2-D gels (see Section 4.5).

\subsection{The rudimentary electroelution apparatus and procedure}

The simple apparatus presented in this paper, in spite of the advance in electric field orientation discussed in Section 4.3 above, is a step backward from the wellengineered, computer controlled HPGE apparatus [4], necessitated by the recent commercial demise of that apparatus. The rudimentary new design (Fig. 1) has the advantage that it can easily be set up in any laboratory at low cost. It is made from common laboratory items (a pipet tip box, a sheet of hard rubber or plastic, a centrifuge tube, electrodes and power supply), except for commercially available glass tubing of a cross-sectional area matching the band and for a micromanipulator.

Any size of gel can be used. However, since the crosssectional area of the elution tube cannot be indefinitely decreased, the larger the number of bands or spots on the electropherogram is, the larger the gel has to be. For that reason, the usefulness of PhastSystem gels as applied in the present study is limited to electro- 
pherograms containing relatively few bands or 2-D spots which are widely separated from one another. If the electroelution apparatus is applied to larger bands than those of the minigels, correspondingly elution tubes with larger cross-sectional area need to be used, and elution time may have to be increased over the $3 \mathrm{~min}$ which were found to be optimal for the minigel bands.

Electroelution into regular arrays (e.g. 11) or grids of electroelution chambers has the advantage of speed and labor saving over the electroelution of single bands proposed here. However, it must fail in the isolation of specific proteins of interest, in resolving closely adjacent bands and in the degree of recovery when there is no overlap in the positions of the band distribution and the electroelution chamber. These defects of the serial and simultaneous electroelution of many bands appear to outweigh its convenience in most practical applications.

The electroelution technique described in the present study has two major shortcomings: (i) it uses protein detection by the reversible Zn-staining method which yields transparent bands against an opaque background. Since these bands are vanishing once the protein-Zn precipitate is solubilized by EDTA for the purpose of electroelution, it is difficult to precisely define the position of the band in terms of its original geometry rather than by the black dot of a marking pen. Clearly, Zn-staining needs to be replaced by the more sensitive and more easily detectable fluorescent labeling such as postlabeling with Sypro Red (Molecular Probes, Eugene, OR, USA, Cat. no. S-6654) and detection by CCD camera [21] or partial prelabeling by FLUOS [8]; (ii) the visual positioning of the elution tube over the band of interest is artful. Positioning under magnification, by magnifying glass or microscope, will be needed. Optimally, the procedure must return to the computer directed positioning of the elution tube over the band of interest, using software similar to that of the HPGE apparatus [4] (see Section 4.5). The basic design (Fig. 1) obviously calls for future maturation along the lines of the 1-D computer controlled HPGE apparatus and its modification for application to 2-D gels.

\subsection{Potential of the apparatus design for sequential band transfer to MS, for application to 2-D PAGE and for automation}

The procedures for the transfer of electroeluted integral protein into MS, including those for SDS removal and protein concentration, applied after electroelution through the design reported here are well-established [5-8]. As pointed out in Section 4.4, the vertical electroelution of proteins by use of the apparatus design depicted in
Fig. 1 is in principle equally applicable to 1-D and 2-D horizontal gels of any size (within the limits of the commercially available dimensions of Gel-Fix). However, the manual electroelution technique is clearly inapplicable to thousands of spots on a 2-D electropherogram due to labor intensiveness. Only when one has a way to detect the spot, or a few spots, of interest, can the manual method presented here be applied to 2-D gels. This is, of course, where the specific immunological detection methods and those using partial sequencing will continue their present role and importance. A sequential electroelution of multiple spots clearly requires an automated procedure. Automated positioning of the electroelution tube on fluorescently detected protein spots can be envisaged as an extension of the hardware and software of the HPGE apparatus [4] to 2-D. Direct interfacing between protein recovery, concentration and $M S$ is obviously a more difficult and remote design problem. Nonetheless, computer-directed electroelution of 2-D spots in extension of the rudimentary design of Fig. 1 appears potentially compatible with automated spot electroelution to a degree to which it is not for a spot transfer procedure requiring gel or blot excision.

\subsection{Sensitivity of protein identification by the procedure}

The apparatus and procedure introduced by the present report has been able to transfer less than 3.7 picomoles of BSA into MS and successful identification of the protein by its mass, as well as to use the remaining 33 picomoles of BSA under a band of SDS-PAGE for establishing the homogeneity of the SDS protein by gel and CZE. The transfer of less than 3.7 picomoles of a protein from a gel electrophoretic band into MS shows an acceptable degree of sensitivity in comparison with conventional methods by which protein on the order of 1 picomole can be transferred from gel electropherograms into MS $[9,10]$. However, the protein load in SDS-PAGE is ten-fold higher if a homogeneity test (re-analysis) of the electroeluate prior to MS is desired for reasons outlined in Section 4.7. The possibility to transfer the integral protein, rather than its proteolytic products, from the gel band has the advantages of allowing for a homogeneity test by reelectrophoresis or gel IEF of the electroeluate prior to MS and for monitoring the removal of SDS from the protein prior to MS while still permitting proteolysis and MS of the proteolytic products.

\subsection{Homogeneity testing prior to MS}

Conventional transfer of proteolysis products from the gel band into MS does not allow one to critically examine the quality of the protein sample to be transferred. Con- 
sequently, the resulting MS analysis of the eluate of a single SDS protein band frequently reveals peptides of more than one protein, making identification of the protein by its peptide map difficult or preventing such identification altogether. The need for a homogeneity test for the protein prior to MS is exacerbated by the fact that even a single band in SDS-PAGE, as demonstrated in Fig. 3, may be composed of multiple proteins differing in surface charge densitiy (e.g. see Fig. 2 of [22]) or size. Ideally, if the resolution of proteins based on mass differences, using SDS-PAGE, and their separation based on differences in surface net charge, using IEF, would be sufficiently effective, a spot on a 2-D electropherogram would be homogeneous, and the peptide map derived from it would unequivocally identify the protein. However, that ideal condition does not exist in reality for a number of reasons: (i) SDS-PAGE may be conducted at a gel concentration which does not provide an optimal resolution between some or all component pairs [23]; (ii) IEF may not have achieved the steady-state for all components at which charge separation is optimal [24]; if the $\mathrm{pH}$ gradient is formed by carrier ampholytes, its cathodic drift may deteriorate the gradient before a steady-state protein pattern ensues [25]; if the $\mathrm{pH}$ gradient is formed by Immobiline, electrofocusing time (Vh) may be insufficient for establishing a constant protein pattern characterized by optimal resolution between components [26]; (iii) band distribution overlap between neighboring protein zones is likely to exist even when the stainable maxima of the distributions appear resolved to the eye (p. 157 of [13]).

Received October 3, 2000

\section{References}

[1] Williams, K. R., Hellman, U., Kobayashi, R., Lane, W. W., Mische, S. M., Speicher, D. W., Techniques in Protein Chemistry VIII, 1997, 99-109.

[2] Shevchenko, A., Wilm, M., Vorm, O. and Mann, M., Anal. Chem. 1996, 68, 850-858.

[3] Rosenfeld, J., Capedevielle, J., Guillemot, J. C., Ferrara, P., Anal. Biochem. 1992, 203, 173-179.
[4] Gombocz, E., Cortez, E., Appl. Theoret. Electrophor. 1995, 4, 197-209.

[5] Gombocz, E., Cortez, E., Yang, L., Rammler, D., Biomed. Prod. 1996, 2, 134-135.

[6] Yefimov, S., Yergey, A. L., Chrambach, A., J. Biochem. Biophys. Methods 2000, 42, 65-78.

[7] Yefimov, S., Sjomeling, C., Yergey, A. L., Li, T., Chrambach, A., Anal. Biochem. 2000, 284, 288-295.

[8] Yefimov, S., Sjomeling C., Yergey A. L., Chrambach, A., Electrophoresis 2001, 22, 999-1003.

[9] Naylor, S., Ji, Q., Johnson, K. L., TomLinson, A. J., Kieper W. C. and Jameson, S. C., Electrophoresis 1998, 19, $2207-$ 2212.

[10] Loo, J. A., Brown, J., Critchley, G., Mitchell, C., Andrews, P. C., Ogorzalek Loo, R. R., Electrophoresis 1999, 20, 743-748.

[11] Andersen, P., Heron, I., J. Immunol. Methods 1993, 161, 29-39.

[12] Triga, D., Pamjav, H., Vellai, T., Fodor, A., Buzas, Zs., Electrophoresis 1999, 20, 1274-1279.

[13] Neuhoff, V., and Maelicke, A. (Eds.) in: Chrambach, A., The Practice of Quantitative Gel Electrophoresis, VCH, Weinheim FRG, 1985, p. 234.

[14] Hjerten, S., J. Chromatogr. 1985, 347, 191-198.

[15] Radko, S. P., Garner, M. M., Caiafa G., Chrambach, A., Anal. Biochem. 1994, 223, 82-87.

[16] Chang, H.-T., Yergey A. L., Chrambach, A., Electrophoresis 2001, 22, 394-398.

[17] Zakharov, S. F., Garner M. M., Chrambach, A., Appl. Theoret. Electrophor. 1995, 5, 25-30.

[18] Aldroubi, A., Zakharov, S. F., Chrambach, A., Appl. Theoret. Electrophor. 1995, 5, 31-34.

[19] Chen, N., Chrambach, A., Electrophoresis 1998, 19, 30963102.

[20] Chen, N., Chrambach, A., Electrophoresis 1997, 18, 781783.

[21] Csapo, Z., Gerstner, A., Sasvari-Szekely, M., Guttman, A., Anal. Chem. 2000, 72, 2519-2525.

[22] Radko, S. P., Stastna, M., Buzas, Zs., Kingsley, D., Chrambach, A., Anal. Biochem. 1999, 273, 146-148.

[23] Rodbard, D., Chrambach, A., Weiss, G. H., in: Allen R. C., Maurer, H. R. (Eds.), Electrophoresis and Isoelectric Focusing on Polyacrylamide Gel, W. de Gruyter, Berlin 1974, pp. 62-105.

[24] Svensson, H., Acta Chem. Scand. 1961, 15, 325-341.

[25] Baumann, G., Chrambach, A., in: Righetti, P. G. (Ed.), Progress in Isoelectric Focusing and Isotachophoresis, Elsevier, Excerpta Medica, North Holland, Amsterdam, 1975, pp. 1323.

[26] Görg, A., Fawcett, J. S., Chrambach, A., in: Chrambach, A., Dunn, M. J., Radola, B. J. (Eds.), Advances of Electrophoresis, 2, VCH, Weinheim, Germany 1988, pp. 1-44. 